\title{
Numerical Investigation on Mechanical Behaviour of Closed-cell Aluminium Foams Using a Representative Volume Element Method
}

\author{
Chengjun Liu' ${ }^{1}$ Y.X. Zhang ${ }^{1}$, Chunhui Yang ${ }^{2^{*}}$ \\ ${ }^{1}$ School of Engineering and Information Technology, UNSW Canberra, Australian Defence Force Academy, ACT 2600, Australia \\ ${ }^{2}$ School of Computing, Engineering and Mathematics, Western Sydney University, Penrith, NSW 2751, Australia
}

\begin{abstract}
In this study, a representative volume element (RVE) method is developed to capture the mechanical behaviour of aluminum foams under compressive loadings. Octadecahedrons are selected for forming cells of a microstructured RVE model to simulate mechanical behaviour of the aluminium foams under quasi-static compressive loadings. A convergence test is conducted to determine an appropriate mesh density. The stress-strain relationship obtained from the numerical simulations is compared to that from experimental study and agreements between these results demonstrate the efficiency of the proposed RVE model. Thereafter failure modes during a compressive loading process of the aluminum foams are also identified and discussed using this RVE model.
\end{abstract}

\section{Introduction}

Closed-cell aluminium foams are very competitive materials for applications in engineering structures where weight and impact resistance are the main concerns, due to their superior mechanical characteristics such as light weight and energy absorption. Typical engineering applications of aluminium foams include automotive vehicles, aeronautical structure, especially on helicopter components, aerospace structures, ship building, railway train and packaging components [1-3]. To facilitate the structural applications of the aluminium foams with an effective and optimal structural design, it is essential to gain an insightful understanding of their mechanical behaviour.

Multiscale modelling is a term for a kind of modelling in which multiple models at different length and/or time scales are developed and employed simultaneously to describe an engineering problem of a material or system. However it is difficult to achieve such a systematic algorithm across both length and time scales. To simplify this complex problem with ignoring the influence from time scale, a systematic framework on multiscale analysis and digital material representation of heterogeneous material [4], which are multiphase and microstructured, has been developed and ustilised to fabricate and evaluate their overall material properties, i.e., porous materials and particle-reinforced composites. It is based on micromechanics and theory of localisation and homogenisation. This digital framework can be employed to cover three-level digital material representations (DMR) across length scales from micro, meso to macro scales: a) unit cell (UC); b) representative volume element (RVE); and c) bulk material. In Yang et al.'s

\footnotetext{
*Corresponding author: r.yang@westernsydney.edu.au
}

study [4], the representative volume element (RVE) models for metal foams were generated using a microstructure generator coded using Matlab with a sphere growth algorithm. Then the finite element modelling was employed to determine their mechanical properties. Other finite element models [5-6] have been also developed and employed to model the metal foams and study their mechanical behaviour, but these models were mainly generated based on a well-known Voronoi tessellation method, which may result in under- or overestimation on mechanical properties due to the randomness and need an averaging post-processing.

Utilising repeating polyhedron cells is a possible way to overcome this flaw and can provide a reasonable estimation without one more post-processing step. Researchers have developed a few finite element models based on tetrakaidecahedron cells for modelling of aluminum foams. Simone and Gibson [7] proposed a model and analysed the effect on stiffness and strength of closed-cell tetrakaidecahedral foams. Song et al. [8] developed a model with tetrakaidecahedron cells to model the dynamic crushing behaviour of aluminium foams. Nonetheless, numerically modelling the mechanical properties of aluminum foams using uniform and closed-cell polyhedrons is still rare.

In this research, a Representative Volume Element (RVE) method is proposed to model the closed-cell aluminium foams focusing on their mechanical behaviour under compressive loading. Octadecahedrons are selected as basis cells to form the RVE model for the aluminium foams. For validation and verification, this microstructured RVE model is employed to model Alporas ${ }^{\circledR}$ foams with a porosity of 0.915 under quasi- 
static compressive loading with a loading rate of 2 $\mathrm{mm} / \mathrm{min}$.

\section{RVE model of Closed-Cell Foams}

This geometric model of the proposed RVE was obtained through cutting 8 octadecahedrons as shown in Figure 1, including one intact octadecahedron wrapped in the center, twelve half-octadecahedrons (four on the top, four in the bottom and another four surrounding the intact cell) and four quarter-octadecahedrons in parallel.

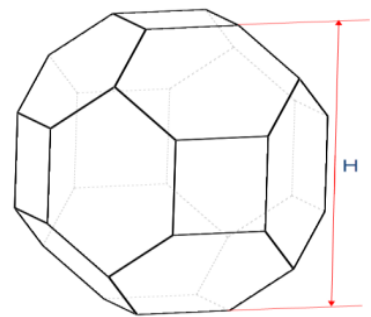

Figure 1. Octadecahedron.

As octadecahedral foams can be accounted as a number of repeated RVEs in the structure, this RVE is considered to represent aluminium foams with uniform and closed octadecahedron cells. The height is used to define the cell size of the octadecahedron and specifically the height $\mathrm{H}$ is fixed to $2.96 \mathrm{~mm}$ in this study while the thickness of the cell $\mathrm{t}$ is $0.034 \mathrm{~mm}$ to guarantee that the relative density of the foam is approximately $8.5 \%$, which is equivalent to the porosity of $91.5 \%$ for the aluminium foam. The geometric model is shown in Figure 2 with the RVE defined as Part 1, and the upper and bottom steel plates defined as Part 2 and Part 3, respectively. Part 3 is defined to support the RVE, while compressive loads are applied to the RVE via moving Part 2.

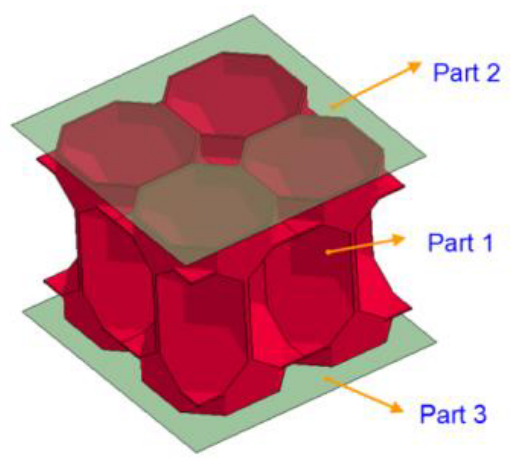

Figure 2. Geometric model of the RVE.

\section{FEA of the RVE model}

This finite element analysis was conducted using commercial finite element analysis software LS-Dyna. Parts 1 and 2 in the FE model were meshed using the Belytschko-Tsay shell elements, with setting a shear correction factor of $5 / 6$, and the number of through shell thickness integration points to 1 . The RVE of foams was meshed using 3D solid element 164 , a fully integrated quadratic 8-node element with nodal rotations. It was divided into eight equal parts as shown in Figure $3 \mathrm{a}$, and each part was divided into hexahedrons (see Figure $3 \mathrm{~b}$ ) and each hexahedron can be meshed using $n \times n$ solid elements.

The material model of aluminum foams used a low cost isotropic plasticity model (Material Type 12). In this model, the strain rate is considered using the CowperSymonds model, which scales the yield stress via a strain rate dependent factor [9]. The steel plates, Part 1 and Part 2 , were both treated as rigid body in the FE model. Material parameters for these two metals are given in Tables 1 and 2, respectively. Two types of contact boundary conditions were defined in this model. One is the mutual contact between different parts, specifically between the RVE and Part 2 or Part 3. The other one is self-contact inside the RVE model resulted from large deformation.

Table 1. Material properties of the aluminium $[2,10]$.

\begin{tabular}{|c|c|c|c|c|}
\hline Density & $\begin{array}{c}\text { Shear } \\
\text { modulus }\end{array}$ & $\begin{array}{c}\text { Yield } \\
\text { stress }\end{array}$ & $\begin{array}{c}\text { Plastic } \\
\text { hardening } \\
\text { modulus }\end{array}$ & $\begin{array}{c}\text { Bulk } \\
\text { modulus }\end{array}$ \\
\hline $\begin{array}{c}2700 \\
\mathrm{~kg} / \mathrm{m}^{3}\end{array}$ & $23.73 \mathrm{GPa}$ & $\begin{array}{c}100 \\
\mathrm{MPa}\end{array}$ & $1.36 \mathrm{GPa}$ & $\begin{array}{c}51.42 \\
\mathrm{GPa}\end{array}$ \\
\hline
\end{tabular}

Table 2. Material properties of the steel [11].

\begin{tabular}{|c|c|c|}
\hline Density & $\begin{array}{c}\text { Young's } \\
\text { Modulus }\end{array}$ & $\begin{array}{c}\text { Poisson's } \\
\text { ratio }\end{array}$ \\
\hline $\begin{array}{c}7850 \\
\mathrm{~kg} / \mathrm{m} 3\end{array}$ & $210 \mathrm{GPa}$ & 0.33 \\
\hline
\end{tabular}

Convergence test was conducted to find out the best value of $\mathrm{n}$ as shown in Figure 3 (b). The compressive force-time curve was computed from the finite element model with different mesh density with $\mathrm{n}$ ranging from 1 to 5. According to Figure 4, converged results are achieved with the mesh density of $n=5$. Therefore $n=5$ was adopted to mesh the RVE model for accuracy and computation efficiency. The finite element mesh using this mesh density is shown in Figure 5.

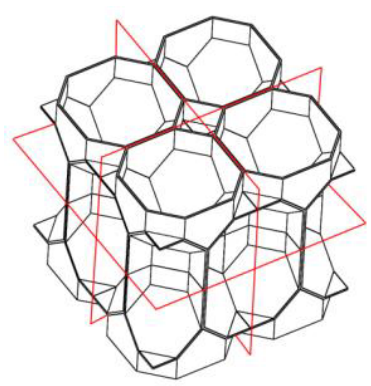

(a)

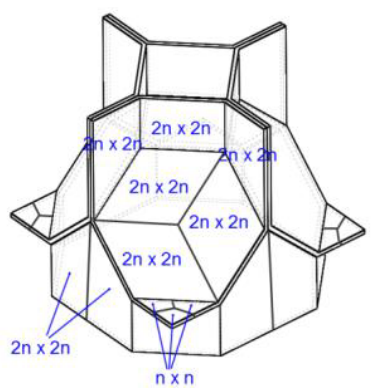

(b)
Figure 3. Mesh refinement of the RVE model. 


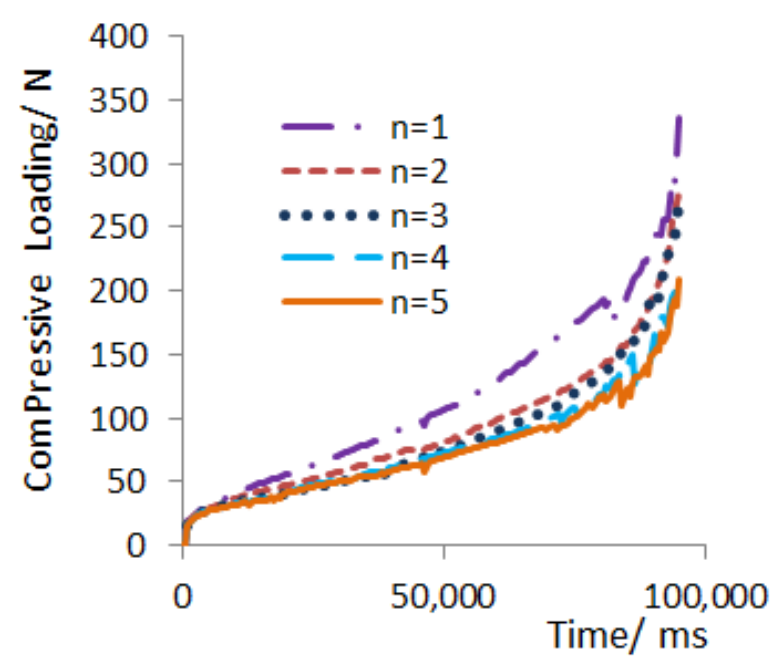

Figure 4. Mesh refinement of the RVE model.

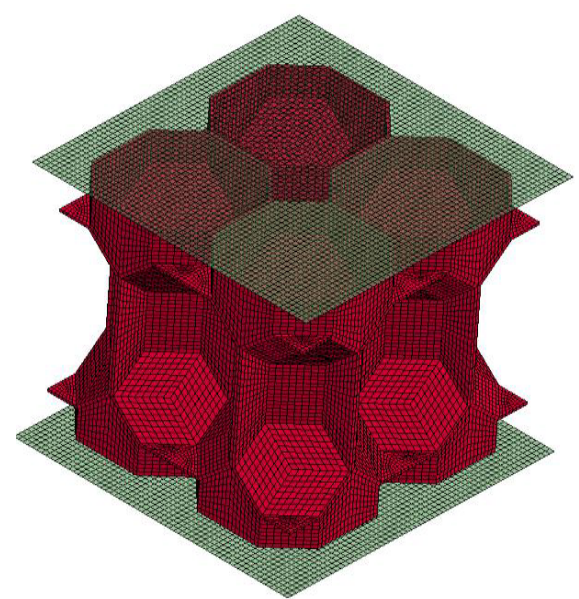

Figure 5. Finite element mesh of the RVE model.

\section{Results and discussion}

As for validation and verification, the stress-strain relations extracted from the RVE model were compared to that from a compression test of equal-porosity Alporas ${ }^{\circledR}$ foams.

Figure 6 compares the compressive stress-strain curves of the aluminium foams obtained from the finite element analysis and the experimental study, respectively [12]. The curve obtained from numerical modelling presents a trend very similar to that from the experiments and the model can capture the hardening behaviour of the material, though there is a discrepancy between the numerical result and experimental data. The RVE model provides lower Young's modulus and yielding stress than those from the experimental work, while the plateau stress in the modelling is higher than that from experimental data. These differences may result from the employing uniform cell and cell size to represent the aluminium foams. However, it shows a probability that the aluminium foams with a uniform cell could present better mechanical properties than aluminium foam with random pore sizes. Overall, the RVE-based finite element model can reflect the mechanical properties of closed-cell aluminium foams.

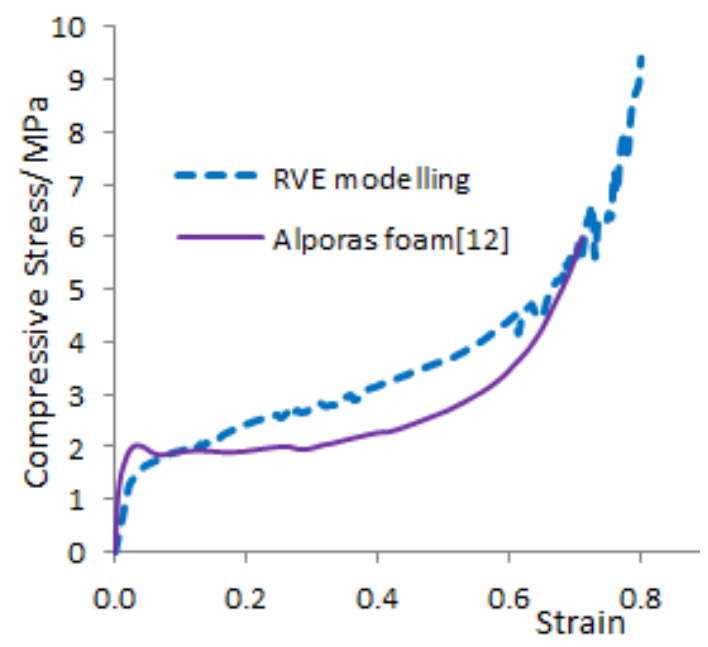

Figure 6. Stress-strain curves of the aluminium foams.

The modelled deformation evolution of the RVE during the compression process is shown in Figure 7. Several failure modes could be observed during the deformation process. The first failure mode is cell wall buckling, which can be clearly found from the cell walls along the compressive loading direction. The second type of the failure modes is stretching, which occurs in the cell walls of both top and bottom semi-cells, in which their normal directions to align with the loading direction. Another failure mode is upsetting in cell walls, where the vertical cell walls become wider and shorter when the compressive loading is applied.

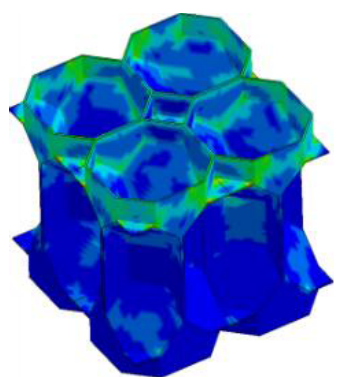

(a)

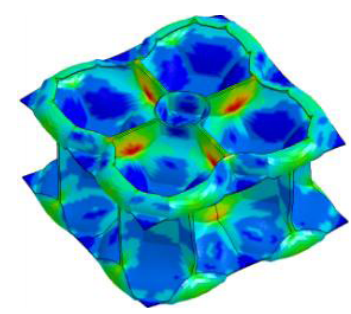

(c)

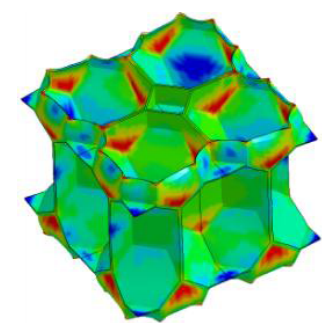

(b)

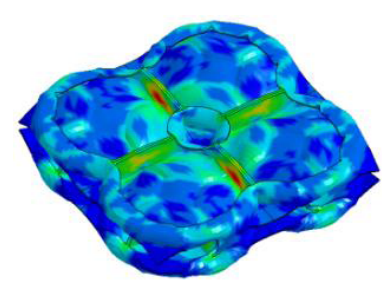

(d)
Figure 7. von Mises stress contour of the RVE during the deformation process. 


\section{Concluding Remarks}

A RVE model based on repeated octadecahedrons has been developed in this study to model the mechanical behaviour of closed-cell aluminum foams numerically. A convergence test has been conducted and the results show that the finite element model converges well.

The compressive stress versus strain curve of the aluminum foams obtained using the proposed RVE model of the Alporas ${ }^{\circledR}$ aluminum foams has been compared to that obtained from experiment. Overall, the RVE-based finite element model can capture the compressive mechanical properties of closed-cell aluminium foam. The deformation process and the failure modes of the RVE during the compressive loading process from the numerical modelling are also presented, in which in total three failure modes have been identified and they are buckling, stretching and upsetting, respectively.

The proposed RVE model can be further extended to model other metal foams considering different porosities and different basis metal materials as part of future work. The strain rate effect could be investigated by using this model as well.

\section{References}

1. L.J. Gibson and M.F. Ashby: Cellular Solids Structure and Properties (Cambridge University Press, 1999).

2. M.F. Ashby, A.G. Evans, N.A. Fleck, L.J. Gibson, J.W. Hutchinson and H.N.G. Wadley: Metal Foams: A Design Guide (Butterworth-Heinemann, Boston 2000).

3. J. Banhart: Prog. Mater. Sci. Vol. 46 (2001) p. 559632.

4. C. Yang, Y. An, M. Tort and P.D. Hodgson: Comput. Mater. Sci. Vol. 81(2014) p. 89-97.

5. K.R. Mangipudi and P.R. Onck: J. Mech. Phys. Solids Vol. 59 (2011) p. 1437-1461.

6. K.R. Mangipudi and P.R. Onck: Acta Mater. Vol. 59 (2011) p. 7356-7367.

7. A.E. Simone and L.J. Gibson: Acta Mater. Vol. 46 (1998) p. 2139-2150.

8. Y. Song, Z. Wang, L. Zhao and J. Luo: Mater. Des.Vol.31 (2010) p. 4281-4289.

9. John O. Hallquist: LS-DYNA Theory manual (March 2006).

10. I. Jeon, K. Katou, T. Sonoda,T. Asahina and K.J. Kang: Mech. Mater. Vol. 41 (2009) p. 60-73.

11. W.H. Hou, F. Zhu, G.X. Lu and D.N. Fang: Int. J. Impact Eng. Vol. 37 (2010) p. 1045-1055.

12. J.K. Hu, G.X. Lu and D. Ruan: Composites Part B Vol. 41 (2010) p. 678-685. 